\title{
Gastric Residual Volume Assessment by Gastric Ultrasound in Fasting Obese Patients: A Comparative Study
}

\author{
Atef Mohammad Khalil (iD ${ }^{1,}$, , Safaa Gaber Ragab (iD) ${ }^{1}$, Joseph Makram Botros (iD) ${ }^{1}$, Hazem Ali Abd-aal ${ }^{1}$ \\ and Maged Labib Boules ${ }^{1}$ \\ ${ }^{1}$ Anesthesia Departement, Faculty of Medicine, Fayoum University Hospital, Egypt \\ "Corresponding author: Anesthesia Departement, Faculty of Medicine, Fayoum University Hospital, Egypt. Email: dr.atef.khalil@gmail.com
}

Received 2020 October 04; Revised 2020 November 19; Accepted 2020 December 28.

\begin{abstract}
Background: Gastric ultrasound is an emerging tool for preoperative evaluation of gastric content and volume.

Objectives: To assess gastric residual volume in normal-weight and obese patients scheduled for elective surgery.

Methods: This prospective observational study was conducted on 100 patients assigned to two groups of 50 patients each. The obese group included patients with body mass index (BMI) of 30 - 40 and American Society of Anesthesiologists (ASA) grade II and those with BMI > 40 and ASA III without other comorbidities; the normal-weight group included patients with normal BMI and ASA I. Gastric volume was predicted in each group using sonographic measurement of antral cross-sectional area (CSA) in semi-sitting and right lateral positions (RLP); the two groups were compared to assess the risk of aspiration for each group preoperatively.

Results: Despite intergroup differences in antral CSA, the sonographically predicted gastric volume was $<1.5 \mathrm{~mL} / \mathrm{kg}$ in both groups in both positions. Both groups were at a low risk for aspiration, and $98 \%$ of the patients showed grade 0 or 1 in antrum assessments, corresponding to an empty antrum and minimal fluid only in the RLP, respectively. Only $2 \%$ of the patients in both groups showed a distended antrum in both positions.

Conclusions: Despite the differences in CSA between obese and normal-weight participants in both positions (obese $>$ normalweight), both groups showed a low predicted gastric residual volume $<1.5 \mathrm{~mL} / \mathrm{kg}$ and were at low risk for aspiration, provided that fasting was initiated at least 8 hours before elective surgery.
\end{abstract}

Keywords: Gastric Residual Volume, Fasting, Obesity, Gastric Ultrasound, Gastric Emptying, Gastric Emptying, Anesthesia

\section{Background}

Delayed gastric emptying is a major risk factor for aspiration of gastric contents into the lungs, a possibly fatal complication during anesthesia (1). Moreover, gastric emptying also influences the systemic availability of orally administered substances, making it doubly important for anesthetists. Delayed gastric emptying can lead to nausea and vomiting, prevent a return to oral feeding, and be responsible for morbidity and mortality (2)

Gastric emptying is influenced by both humoral and neural factors (3). The volume and composition of gastric food act as major determinants of the rate of gastric emptying (4). Nevertheless, studies on the effect of body weight on gastric emptying have yielded inconsistent findings (5). The application of fasting guidelines provided by the American Society of Anesthesiologists (ASA) ensures that the stomach is emptied before anesthesia induction, which is the primary method to prevent aspiration (6).
However, these guidelines cannot be applied in urgent procedures, or patients with morbidities associated with delayed gastric emptying (7).

Previous studies have reported the use of ultrasound imaging preoperatively for bedside assessment of gastric content and volume in non-fasting, diabetic, severely obese, and intensive care patients (8-10). Sayyadi et al (9). had employed ultrasonography to study the effect of metoclopramide on gastrointestinal tract (GIT) motility in patients with incomplete fasting.

\section{Objectives}

This study was conducted to assess gastric residual volume with focused abdominal sonography in normalweight and obese patients scheduled for elective surgery. 


\section{Methods}

This prospective observational study was conducted between July 2019 and June 2020 at our university hospital after receiving approval from the local institutional ethics committee and registering the clinical trial. Eligible participants were provided a detailed study explanation and provided informed consent before enrollment. The study protocol adhered to CONSORT guidelines.

The study population consisted of a total of 100 obese patients (body mass index $(\mathrm{BMI})>30$ ) and normal-weight patients with BMI between 18 and 24.9 scheduled to undergo elective surgery. We excluded patients meeting any of the following criteria: pregnancy, renal failure, liver cell failure, or a history of upper GIT morbidity or upper abdominal surgery, diabetes, opium addiction, and other conditions or usage of drugs affecting gastric motility. All patients enrolled in the study were $>20$ years old. The participants were assigned to two groups of 50 patients each. The obese group included participants with BMI between $30-40$ and ASA II, and those with BMI > 40 and ASA III without other comorbidities; the second group consisted of normal-weight participants with ASA I. The participants underwent an 8-h preoperative overnight fast after a light meal.

Focused abdominal sonography was performed in the semi-sitting and right lateral position (RLP). A trained physician used the Mindray curved transducer at a low frequency (2 - $5 \mathrm{MHz}$ ) for abdominal examination. A sweep of the probe from the left to right subcostal margins determined the antrum to be located within the epigastrium. For this assessment, the left liver lobe and the descending abdominal aorta were used as anterior and posterior landmarks, respectively. Based on antral shape and contents, a flat antrum with juxtaposed anterior and posterior walls was considered empty; a distended antrum with hypoechoic contents and thin walls was considered fluidcontaining; and a distended antrum with contents showing mixed echogenicity was considered solid-containing. The antral cross-sectional area (CSA) was measured using the equation:

$C S A=\pi \frac{[D 1 \times D 2]}{4}$

(D1 and D2: two antral dimensions).

Gastric residual volume in each patient position was calculated using the following equations: gastric residual volume $(\mathrm{mL})=215+57 \log \mathrm{CSA}\left(\mathrm{cm}^{2}\right)-0.78$ age (year) -0.16 height $(\mathrm{cm})$ - 0.25 weight $(\mathrm{kg})-0.80$ ASA (Bouvet and colleagues' equation for semi-sitting position); gastric residual volume $(\mathrm{mL})=27.0+14.6 \times$ rightlateral CSA - $1.28 \times$ age (Perlas and colleagues' equation for RLP).

The aspiration risk was categorized using the system proposed by Ven de Putte and Perlas. In this system, the aspiration risk is classified as (a) patients with empty antrum and gastric residual volume $<1.5 \mathrm{~mL} / \mathrm{kg}$ : low risk; (b) patients with solid contents or gastric residual volume $>1.5$ $\mathrm{mL} / \mathrm{kg}$ : high risk.

An 18-F nasogastric tube was inserted after anesthesia induction and endotracheal intubation. Gastric contents were aspirated by gentle suction with a $50 \mathrm{~mL}$ syringe with a synchronous epigastric massage. The primary outcome was gastric residual volume in the semi-sitting position. Secondary outcomes included the grade of aspiration risk (high and low), fluid volume aspirated from the nasogastric tube after anesthesia induction, gastric residual volume in the RLP, and antral CSA in both semi-sitting and RLP. Antrum findings were graded as empty antrum: grade 0 ; minimal fluid in the RLP only: grade 1 ; distention of antrum in both semi-sitting position and RLP: grade 2 .

\subsection{Statistical Analysis}

Statistical Package for the Social Sciences (version 22, SPSS Inc, USA) was used to organize, tabulate, and statistically analyze the collected data. Numerical variables were presented as median and interquartile range (IQR) since they were not normally distributed; the test of significance was determined using the Mann-Whitney U test. Numbers and percentages were used to represent qualitative data; the test of significance was determined using the chisquared test. A two-tailed P-value $<0.05$ was considered statistically significant.

\subsection{Sample Size}

The G-Power@ software version 3.1.7 was used to calculate the sample size with an effect size of 0.57 , two-sided (two tails) type I error of 0.05 , and power of $80 \%$. Each group was required to include at least 50 participants.

\section{Results}

Table 1 shows that age and sex were not significantly different between groups (normal-weight and obese patients; $\mathrm{P}=0.159$ ), but ASA classification showed a significant difference since $100 \%$ of patients in the normal-weight group had ASA I status, while $86 \%$ and $14 \%$ of the patients in the obese group had ASA II and ASA III status, respectively, regarding obesity only without other comorbidities $(\mathrm{P}<$ 0.0001). 


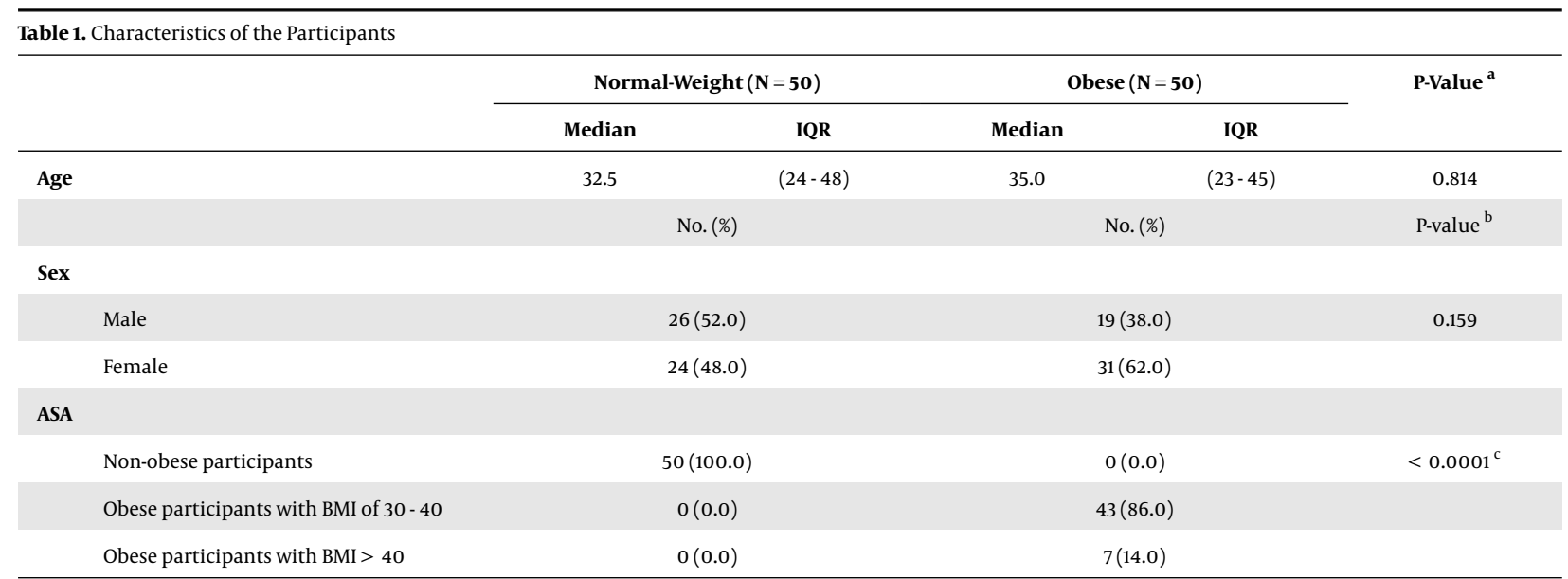

Abbreviations: IQR, interquartile range; ASA, American Society of Anesthesiologists.

aann-Whitney U test.

${ }^{\mathrm{b}}$ Chi-square test.

${ }^{\mathrm{c}}$ Significant.

Table 2 shows that unlike weight and BMI, which showed significant differences between groups $(\mathrm{P}<$ 0.0001); height was not significantly different between obese and normal-weight patients.

Table 3 shows that the gastric volume was not significantly different between groups in ultrasound measurements performed in the semi-sitting position while fasting; however, the two groups showed significant differences in the D1 (craniocaudal) $(\mathrm{P}=0.011)$ and D2 (anteroposterior $)(\mathrm{P}<0.0001)$ diameters, as well as the antral CSA, which was a derived value $(\mathrm{P}<0.0001)$.

Table 4 shows significant intergroup differences in antral diameters D1 $(\mathrm{P}=0.002)$ and $\mathrm{D} 2(\mathrm{P}<0.0001)$, as well as antral CSA and gastric residual volume $(\mathrm{P}<0.0001)$ in abdominal ultrasound assessments in the RLP.

Table 5 shows that $52 \%$ and $58 \%$ of participants in the normal-weight and obese groups, respectively, had an empty antrum (grade 0$)(\mathrm{P}=0.830)$. On the other hand, $46 \%$ and $40 \%$ of participants in the normal-weight and obese groups, respectively, had minimal antral fluid only in RLP (grade 1), with no significant difference between groups (P $=0.830$ ). Moreover, $2 \%$ of the participants in both groups showed a distended antrum (grade 2 ) in both RLPand semisitting positions; however, the difference was not significant $(\mathrm{P}=0.830)$.

Table 6 shows no significant difference in the aspiration risk between normal-weight and obese patients fasting for $8 \mathrm{~h}$ before elective surgery $(\mathrm{P}=1.000)$. Only $2 \%$ of participants in both groups were at a high risk for aspiration. Table 7 shows that nasogastric tube insertion after anesthesia induction for measurement of gastric residual volume did not differ between the groups $(\mathrm{P}=0.806)$.

\section{Discussion}

Abdominal ultrasound has been used to assess gastric content and volume in humans, but the difference between preoperative gastric antral diameters of normalweight and obese patients undergoing elective surgeries have not been investigated. Assessment of the gastric volume and content greatly facilitated evaluation of the risk of aspiration of gastric content in the two groups (11). Aspiration pneumonia due to gastric content aspiration during anesthesia is a preventable complication, accounting for up to $9 \%$ of all deaths associated with anesthesia, with several coexisting mechanisms contributing to this phenomenon. The critical gastric fluid volume that may increase aspiration risk in patients during anesthesia remains unknown; however, several studies show that healthy fasting patients with residual gastric volumes larger than previously assumed (up to $1.5 \mathrm{~mL} / \mathrm{kg}$ ) are not at an increased risk of aspiration (12). The adoption of universal "fasting guidelines" before elective surgery plays an important role in mitigating risk by limiting gastric contents during the immediate perioperative period. However, these guidelines cannot be applied to patients in specific physiologic states such as pregnancy, those undergoing emergent or urgent surgery, or those with coexisting medical conditions (13).

According to Bouvet et al.(14) the gastric contents of 65 in 66 (98\%) patients could be identified in the semi-sitting position, and gastric antrum imaging was successful in 


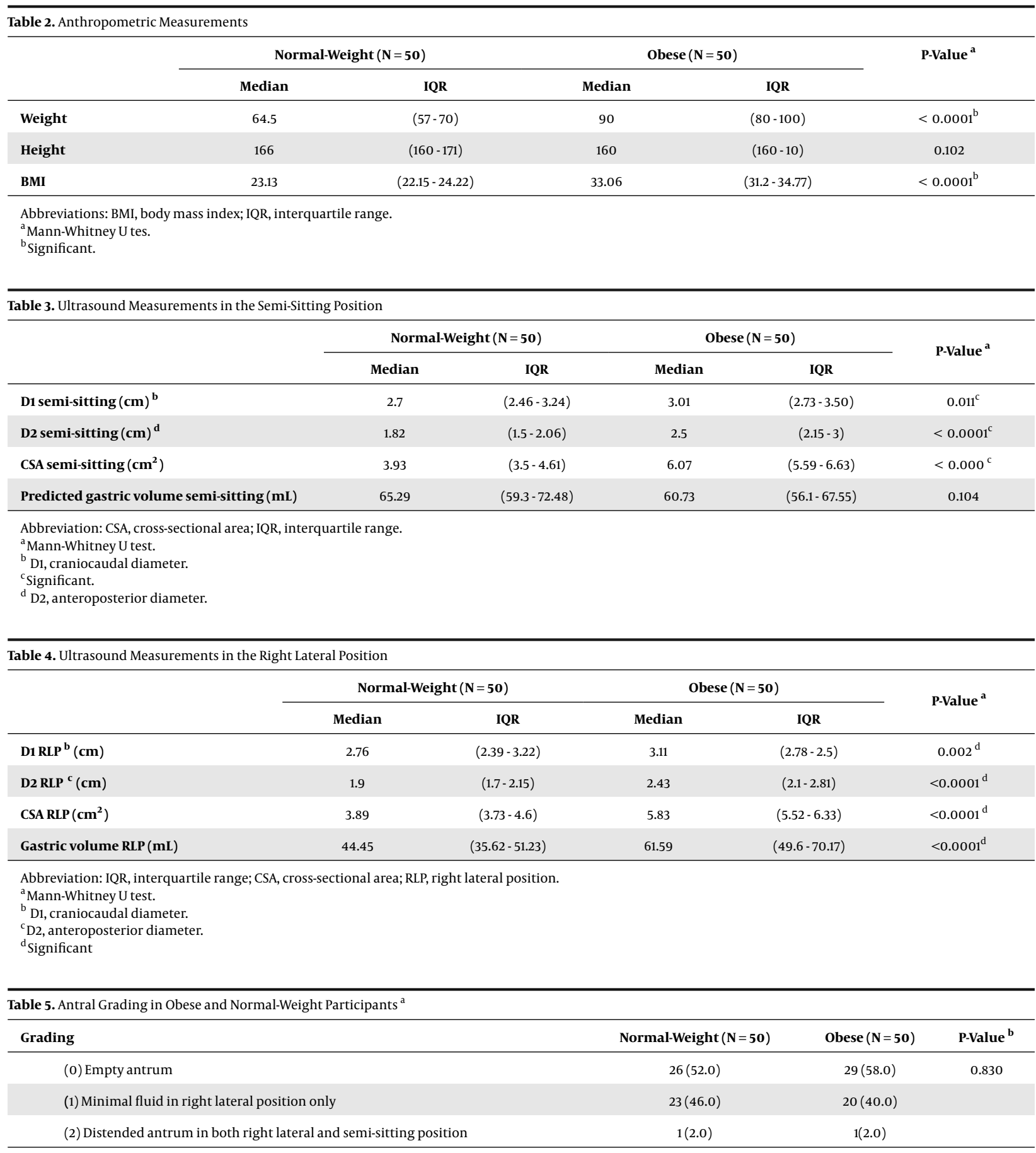

${ }^{\mathrm{a}}$ Values are expressed as No. (\%)

${ }^{\mathrm{b}}$ Chi-square test.

95\% and $90 \%$ of the subjects in the RLP and supine positions, respectively, by Van de Putte et al (15). In the RLP position, a $100 \%$ success rate was reported by Perlas et al (16). The present study could measure the antral area in all 100 patients in the RLP and semi-sitting positions, consistent with R. Kruisselbrink et al. (7), who identified the antrum in all 38 subjects included in the final analysis of their study. This is not surprising because irrespective of 


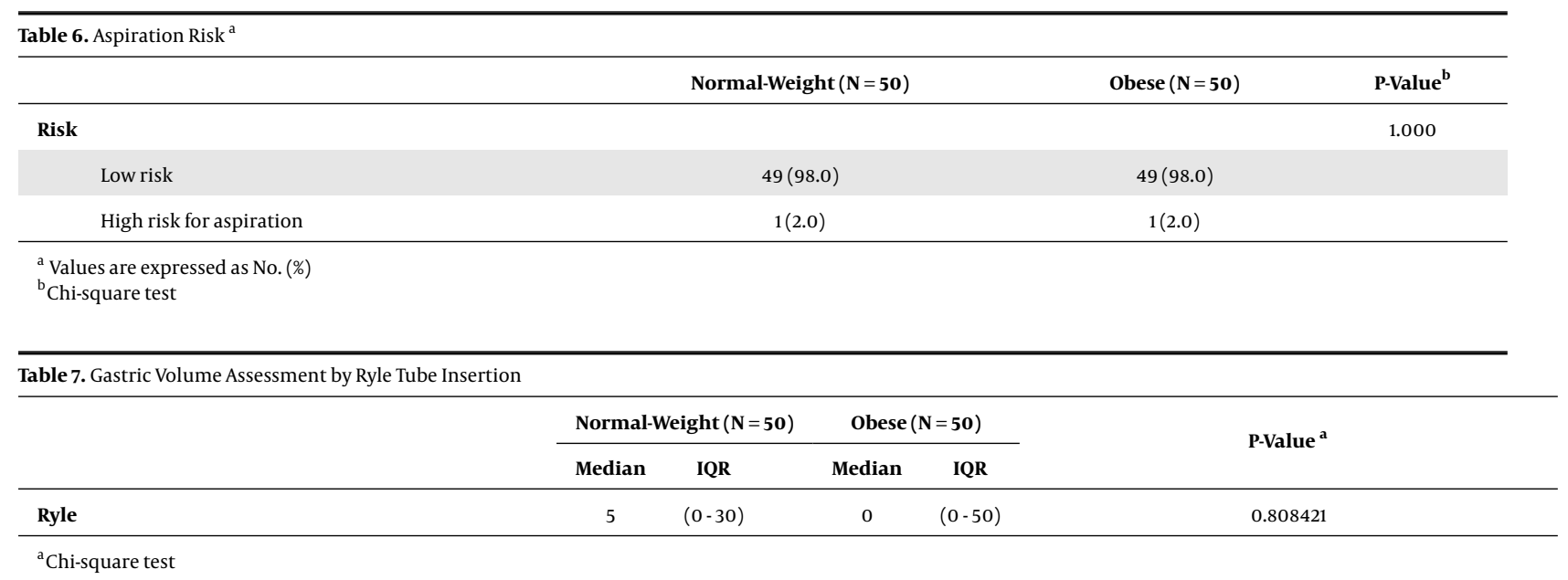

gas contents in the fundus, the antrum was reportedly visible in each patient before and after ingestion of liquids or solid foods. (17). Compared to the supine position, scanning in the RLP or semi-sitting position has been shown to improve sensitivity for detection of small gastric volumes, since a substantial portion of gastric content moves favorably to the more dependent antrum (18).

In our study, no patient showed solid content in the stomach, similar to the findings reported by Van de Putte pet al (15). For an elective surgical fasting population, there was no significant difference between obese and normalweight patients in antral grading since most of the normalweight and obese patients had antral grade 0 (52\% and 58\%, respectively), and a lower proportion had antral grade 1 ( $46 \%$ and $40 \%$, respectively). The remaining (2\%) patients in both groups were classified as antral grade 2 with fluid content and a clearly distended gastric antrum visible in both semi-sitting and RLP positions. In the semi-sitting position, the predicted gastric volume was $65 \pm 6 \mathrm{~mL}$ and 60 $\pm 5 \mathrm{~mL}$ in the normal-weight and obese patients, respectively; in the RLP, it was $44 \pm 8 \mathrm{~mL}$ and $61 \pm 10 \mathrm{~mL}$ in the normal-weight and obese patients, respectively.

Perlas et al. described the findings for 200 patients, with most (193 of $200 ; 96.5 \%$ ) classified as grade 0 or 1 (grade $0,43 \%$; grade $1,53.5 \%$ ). The remaining seven (3.5\%) patients were classified as grade 2 with fluid content and a distended gastric antrum visible in both supine positions and RLP (16). In contrast, Van de Putte et al. graded the antrum in 53 of 60 (88.3\%) patients with a BMI ranging between 35.1 and $68.7 \mathrm{~kg} / \mathrm{m}^{2}$ (95\% CI, $0.77-0.95$ ). Among these, 21 (39.6\%) patients were classified as grade 0, 29 (54.7\%) as grade 1 , and 3 (5.7\%) as grade 2 antrum (15). The predicted volumes for the two groups (grade 0 and 1 ) in the study by Perlas et al. were 0 and $16 \pm 36 \mathrm{~mL}$, respectively, and the corresponding value was $180 \pm 83 \mathrm{~mL}$ for the third group (grade 2). Further, while one patient in their study had a gastric content regurgitation episode during anesthesia emergence, the current study had no such occurrence.

Antral grade showed no significant relationship with age, gender, weight, and BMI in our study, unlike the findings by Van de Putte et al (15). They compared severely obese and non-obese individuals and observed larger baseline gastric volumes $(\mathrm{P}<0.001)$ and a larger antral CSA in severely obese individuals. Similarly, in a previous study by Wong et al. on pregnant patients, a slightly larger fasting CSA was reported in obese versus non-obese patients $\left(5.2 \pm 2.1 \mathrm{~cm}^{2}\right.$ vs. $\left.4 \pm 2.5 \mathrm{~cm}^{2}\right)$ (19). In our study, the CSA of obese patients (median $=6.07 \mathrm{~cm}^{2}$ ) was larger than that of normal-weight patients (median $=3.93 \mathrm{~cm}^{2}$ ) in the semisitting position $(\mathrm{P}<0.0001)$, while gastric volume was not significantly different $(\mathrm{P}=0.104)$. Measurements in the RLP showed significant intergroup differences in CSA (median $=3.89 \mathrm{~cm}^{2}$ and $5.83 \mathrm{~cm}^{2}$ in the normal-weight and obese groups, respectively) and gastric volumes (median $=44.45$ $\mathrm{mL}$ and $61.59 \mathrm{~mL}$, in the normal-weight and obese groups, respectively) $(\mathrm{P}<0.0001)$. According to a study by Philips $S$ et al., the upper limit of residual gastric fluid was between 75 and $130 \mathrm{~mL}$ after aspiration of gastric fluid through a nasogastric tube immediately after induction (20). however, in our study, the upper limit of gastric fluid aspiration through nasogastric tube was $50 \mathrm{~mL}$. The data collected in the present study showed that fasting for 8 hours before elective surgery resulted in a low aspiration risk in $98 \%$ of the participants in both groups since both groups had a gastric residual volume $<1.5 \mathrm{~mL} / \mathrm{Kg}$. Only $2 \%$ of the patients had a high risk of aspiration in both groups despite differences in their antral CSA in the RLP and semi-sitting positions on abdominal ultrasound. One of the limitations 
of our study was that a single operator performed all measurements; according to a recent study describing learning curves in patients with BMI of $25 \pm 3 \mathrm{~kg} / \mathrm{m}^{2}$, obtaining a $95 \%$ success rate with qualitative assessment of gastric content required a mean of 33 examinations (21). In this context, 3-dimensional ultrasonography may be a promising modality to accurately assess gastric volume; however, the data obtained with this method remain preliminary (22).

Third, since the stomach is a dynamic organ that is constantly emptying, the volume at the time of ultrasound assessment may have been higher than at the time of suctioning; the observed "overestimation may be explained through this phenomenon.

\subsection{Conclusion}

Despite the differences in CSA of obese and normalweight individuals in gastric sonography measurements performed in both semi-sitting and RLP (obese $>$ normalweight), both groups showed low predicted gastric residual volumes of $<1.5 \mathrm{~mL} / \mathrm{kg}$. Both groups had low aspiration risk, with most patients in both groups categorized as grade 0 and 1, indicating an empty antrum and an antrum filled with fluid in the RLP alone, respectively, after fasting for at least $8 \mathrm{~h}$ before elective surgery.

\section{Footnotes}

Authors' Contribution: The study was designed by AM and JM. ML enrolled patients and was responsible for the integrity of the data. Analysis was conducted by SG, AM, and JM. ML, JM, and HA wrote the first draft of the manuscript. SG and AM searched the database. JM, ML, and SG all contributed to editing the manuscript.

Clinical Trial Registration Code: NCT04544371.

Conflict of Interests: The authors declare that they have no competing interests.

Ethical Approval: This prospective observational study was conducted between July 2019 and June 2020 at our university hospital after receiving approval from the local institutional ethics committee (M420).

Funding/Support: This work was funded in part by Fayoum University Hospitals, Fayoum, Egypt, and by the authors personal resources.

Informed Consent: Eligible participants were provided a detailed study explanation and provided informed consent before enrollment

\section{References}

1. Olsson GL, Hallen B, Hambraeus-Jonzon K. Aspiration during anaesthesia: a computer-aided study of 185,358 anaesthetics. Acta Anaesthesiol Scand. 1986;30(1):84-92. doi: 10.1111/j.1399-6576.1986.tb02373.x. [PubMed: 3754372].

2. Nimmo WS. Effect of anaesthesia on gastric motility and emptying. Br J Anaesth. 1984;56(1):29-36. doi: 10.1093/bja/56.1.29. [PubMed: 6362691].

3. Grundy D. Vagal control of gastrointestinal function. Baillieres Clin Gastroenterol. 1988;2(1):23-43. doi: 10.1016/0950-3528(88)90019-X. [PubMed: 2838109].

4. Velchik MG, Reynolds JC, Alavi A. The effect of meal energy content on gastric emptying. J Nucl Med.1989;30(6):1106-10. [PubMed: 2738691].

5. Petring OU, Blake DW. Gastric emptying in adults: an overview related to anaesthesia. Anaesth Intensive Care. 1993;21(6):774-81. doi: 10.1177/0310057X9302100605. [PubMed: 8122733].

6. Apfelbaum JL, Caplan RA, Connis RT, Epstein BS, Nickinovich DG Warner MA. American Society of Anesthesiologists Committee: Practice guidelines for preoperative fasting and the use of pharmacologic agents to reduce the risk of pulmonary aspiration: application to healthy patients undergoing elective procedures: an updated report by the American Society of Anesthesiologists Committee on Standards and Practice Parameters. Anesthesiology. 2011;114(3):495-511. doi: 10.1097/ALN.ob013e3181fcbfd9. [PubMed: 21307770].

7. Kruisselbrink R, Arzola C, Jackson T, Okrainec A, Chan V, Perlas A. Ultrasound assessment of gastric volume in severely obese individuals: a validation study. $\mathrm{Br} J$ Anaesth. 2017;118(1):77-82. doi: 10.1093/bja/aew400. [PubMed: 28039244].

8. Perlas A, Chan VW, Lupu CM, Mitsakakis N, Hanbidge A. Ultrasound assessment of gastric content and volume. Anesthesiology. 2009;111(1):82-9. doi: 10.1097|ALN.0b013e3181a97250. [PubMed 19512861]

9. Sayyadi S, Raoufi M, Arhami Dolatabadi A, Rostami M, Memary E. Ultrasonographic assessment of metoclopramide administration effectiveness in accelerating gastric emptying before urgent surgery in patients with insufficient NPO iime. Anesth Pain Med. 2020;10(5). e107331. doi: 10.5812/aapm.107331.

10. Sabry R, Hasanin A, Refaat S, Abdel Raouf S, Abdallah AS, Helmy N Evaluation of gastric residual volume in fasting diabetic patients using gastric ultrasound. Acta Anaesthesiol Scand. 2019;63(5):615-9. doi 10.1111/aas.13315. [PubMed: 30609007].

11. Van de Putte P, Perlas A. Ultrasound assessment of gastric content and volume. $\mathrm{Br}$ J Anaesth. 2014;113(1):12-22. doi: 10.1093/bja/aeu151. [PubMed: 24893784].

12. Hausel J, Nygren J, Lagerkranser M, Hellstrom PM, Hammarqvist F, Almstrom C, et al. A carbohydrate-rich drink reduces preoperative discomfort in elective surgery patients. Anesth Analg. 2001;93(5):134450. doi: 10.1097/00000539-200111000-00063. [PubMed: 11682427].

13. Neelakanta G, Chikyarappa A. A review of patients with pulmonary aspiration of gastric contents during anesthesia reported to the Departmental Quality Assurance Committee. J Clin Anesth. 2006;18(2):102-7. doi:10.1016/j.jclinane.2005.07.002. [PubMed:16563326].

14. Bouvet L, Miquel A, Chassard D, Boselli E, Allaouchiche B, Benhamou D. Could a single standardized ultrasonographic measurement of antral area be of interest for assessing gastric contents? A preliminary report. Eur J Anaesthesiol. 2009;26(12):1015-9. doi 10.1097/EJA.0b013e32833161fd. [PubMed: 19707146].

15. Van de Putte P, Perlas A. Gastric sonography in the severely obese surgical patient: a feasibility study. Anesth Analg. 2014;119(5):1105-10. doi: 10.1213/ANE.0000000000000373. [PubMed: 25054584].

16. Perlas A, Davis L, Khan M, Mitsakakis N, Chan VW. Gastric sonography in the fasted surgical patient: a prospective descriptive study. Anesth Analg. 2011;113(1):93-7. doi: 10.1213/ANE.0b013e31821b98c0. [PubMed: 21596885]. 
17. Bolondi L, Bortolotti M, Santi V, Calletti T, Gaiani S, Labo G. Measurement of gastric emptying time by real-time ultrasonography. Gastroenterology. 1985;89(4):752-9. doi: 10.1016/0016-5085(85)905694. [PubMed: 3896910].

18. Schmitz A, Thomas S, Melanie F, Rabia L, Klaghofer R, Weiss M, et al. Ultrasonographic gastric antral area and gastric contents volume in children. Paediatr Anaesth. 2012;22(2):144-9. doi: 10.1111/j.14609592.2011.03718.x. [PubMed: 21999211].

19. Wong CA, McCarthy RJ, Fitzgerald PC, Raikoff K, Avram MJ. Gastric emptying of water in obese pregnant women at term. Anesth Analg. 2007;105(3):751-5. doi: 10.1213/01.ane.0000278136.98611.d6. [PubMed 17717235].

20. Phillips S, Hutchinson S, Davidson T. Preoperative drinking does not affect gastric contents. Br J Anaesth. 1993;70(1):6-9. doi: 10.1093/bja/70.1.6. [PubMed: 8431336].

21. Arzola C, Carvalho JC, Cubillos J, Ye XY, Perlas A. Anesthesiologists' learning curves for bedside qualitative ultrasound assessment of gastric content: a cohort study. Can J Anaesth. 2013;60(8):771-9. doi: 10.1007/s12630-013-9974-y. [PubMed: 23703533].

22. Manini ML, Burton DD, Meixner DD, Eckert DJ, Callstrom M, Schmit G, et al. Feasibility and application of 3-dimensional ultrasound for measurement of gastric volumes in healthy adults and adolescents. I Pediatr Gastroenterol Nutr. 2009;48(3):287-93. doi: 10.1097/mpg.ob013e318189694f. [PubMed: 19274782]. [PubMed Central: PMC2654599]. 